\title{
A possible solution for the repair of a futuristic Mars suit using nanorobots Gautham Dharuman
}

Dept. of Electrical Engg., National Institute of Technology, Warangal, India harshavardhangautham@gmail.com

\begin{abstract}
Manned mission to Mars will be inevitable due to limitations of the robotic missions. A design is proposed wherein a colony of the Mars suit repair nanorobots will be present above the gas layer (usually oxygen at an average pressure of $560 \mathrm{Kpa}$ ) and beneath the layer of the suit exposed to the external environment. Since the pressure on the martian surface is very low $(0.7$ Kpa on an average), when a region of the suit is damaged, the nanorobots will immediately rush to that region due to the pressure difference. Nanorobots quickly seal breaches by interlocking with one another in successive waves until the damage is sealed. The propulsion of the nanorobots to the region of damage and the interlocking mechanism will follow a set of behavioral rules based on a behavioral strategy to enable a
\end{abstract} coordinated action so that the breach is sealed in a fast and efficient manner.

Keywords: Mars manned mission, suit repair, Flagellar motors, swarm intelligence, Van der waals interactions. Introduction

Manned mission to Mars will be inevitable due to the limitations of the robotic missions. Surface exploration is the key to the mission involving long durations of surface activity (Hoffman et al., 1997) including activities such as digging, climbing over rocks and mountains, and hiking long distances.
Fig 1. Distal margin of Bonneville's ejecta deposit (black dashed line) from "Middle Ground" hollow (Grant et al., 2004)

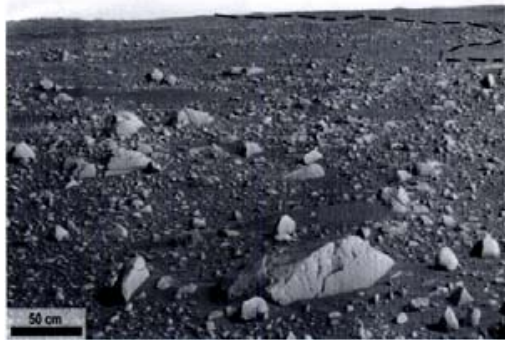

perform specific tasks may enable improvements for nanotechnology automation (Cavalcanti, 2003; Zhang et al., 2004). The next level of challenge for nanotechnology development may address intelligent control system with device manufacturing and automation in a broad sense (Toth-Fejel, 2000; Li et al., 2004). One such application of the nanorobots with respect to the manned Mars mission (Leary et al., 2006) is to employ as intelligent systems for real time sealing of the damages on the mars suit.

\section{Potential dangers}

To explore for evidences which could prove the aqueous sedimentation events of the past (Grant et al., 2004; Squyres et al., 2004), the astronauts will have to undertake surface exploration activities will continue for a period 18 to 20 months (Leary et al., 2006), during which the astronauts are likely to be exposed to the following dangers:

- Gamma rays from newborn black holes and galactic cosmic rays (GCRs) from distant exploding stars Carslaw et al., 2002; Yu, 2002).

- Solar flares with high energy protons (Svensmark et al., 1997).

- Dust storms containing rocks traveling at high speeds (Milam et al., 2003; Grant et al., 2004; Greeley et al., 2004).

From the Fig. 1 one can infer that rocks in the size range of $50 \mathrm{~cm}$ are also Such strenuous activities on a hostile terrain are likely to damage the suit thereby posing a threat to the safety of astronauts involved in surface exploration.

Nanorobots with sizes comparable to bacteria could provide many novel capabilities through their ability to sense and act in microscopic environments. Particularly interesting are bio-engineering applications (Couvreur \& Vauthier, 2006; Leary et al., 2006) where nanorobots and nanoscale-structured materials inside the body provide significant improvements in diagnosis \& treatment of disease (Morris, 2001; Freitas Jr., 2003; Freitas Jr., 1997). The rapid progress in building nanoscale devices should enable a wide range of capabilities (Patel et al., 2006). For example, ongoing development of molecularscale electronics (Howard, 1997; Montemagno, 1999), sensors and motors provide components to enable nanorobots (Cavalcanti et al., 2005; Cavalcanti et al., 2006). Demonstrations of programmable bacteria (Weiss \& Knight Jr., 2000) can produce computation capability for nanodevices. Nano-build hardware integrated systems has been demonstrated and manufacturing techniques are advancing gradually (Cavalcanti et al., 2006). The development of nanosystems for control of nanorobots to present on the Martian soil. These rocks pose huge danger to the astronauts on the field when they gather large momentums in the martian dust storms. All these factors could cause severe damage to the mars suit thereby putting the astronaut's life at risk.

Failure modes: The following failure modes are possible when the mars suit is damaged:

- Primary failure: damage extends to the pressureretaining layer causing gas loss due to the large pressure difference. This might result in the fatal death of the astronaut if not immediately rushed to a pressurized habitat.

- Secondary failure: damage doesn't extend to the pressure-retaining layer but should be sealed before extending to the gas bladder.

The failure modes have been explained in detail in (Benjamin, 2000) in which a similar solution has been attempted for the mars suit repair. Further classification of failure modes based on the types of damage as follows:

- Tears- minimal loss of suit material is associated with this failure. A tear might be caused while using a sharp implement, stretching suit material significantly past its elastic range, or by scraping on a sharp rock. 
- Puncture- significant damage is inflicted on the suit. Could be caused by high velocity rocks carried by dust storms.

- Abrasion- gradual degradation of the suit in the regions of the excessive stretching. Not a severe damage but in the long run could penetrate to the gas layer.

The protection of the astronaut in the field depends entirely on the Mars suit. Since the probability of Mars suit damage in the field is not negligible due to the factors discussed, it is necessary to develop a real time intelligent system which can temporarily seal the damage. The repair solution must provide the astronaut enough time to get back to the pressurized habitat safely.

Mechanism of repair

The repair mechanism proposed in this paper could provide a possible, cost and time effective solution to the problem of Mars suit repair in real time. The architecture for the repair sequence involving a number of processes was decided taking into account the vital aspects of the Martian environmental conditions.

Martian environmental conditions

Atmosphere: The atmosphere of Mars is composed primarily of carbon dioxide with small amounts of other gases (Hamilton, 2001). The six most common components of the atmosphere are: Carbon Dioxide $\left(\mathrm{CO}_{2}\right)$ : 95.32\%; Nitrogen $\left(\mathrm{N}_{2}\right): 2.7 \%$; Argon $(\mathrm{Ar}): 1.6 \%$; Oxygen $\left(\mathrm{O}_{2}\right)$ : $0.13 \%$; Water $\left(\mathrm{H}_{2} \mathrm{O}\right)$ : $0.03 \%$; Neon $(\mathrm{Ne})$ : $0.00025 \%$. The Martian air contains only about $1 / 1000$ as much water as our air. Therefore, nanorobots which can utilize the carbon-di-oxide for energy production should be developed.

Temperature: The average recorded temperature on Mars is $-63^{\circ} \mathrm{C}\left(-81^{\circ} \mathrm{F}\right)$ with a maximum temperature of $20^{\circ} \mathrm{C}\left(68^{\circ} \mathrm{F}\right)$ and a minimum of $-140{ }^{\circ} \mathrm{C}\left(-220^{\circ} \mathrm{F}\right)$ (Hamilton, 2001). Therefore, the operating temperature range for the nanorobots has to be between $-140{ }^{\circ} \mathrm{C}$ to 20 ${ }^{\circ} \mathrm{C}$. With the use of unique thermally insulating skins we could narrow down this range further but need to find the exact figures.

Pressure: Barometric pressure varies at each landing site on Mars on a semiannual basis. $\mathrm{CO}_{2}$, the major constituent of the atmosphere, freezes out to form an immense polar cap, alternately at each pole (Hamilton, 2001). $\mathrm{CO}_{2}$ forms a great cover of snow and then evaporates again with the coming of spring in each hemisphere. When the southern cap was largest, the mean daily pressure observed by Viking Lander 1 was: as low as 6.8 millibars $(\sim 0.7 \mathrm{Kpa})$; at other times of the year it was as high as 9.0 millibars $(\sim 0.9 \mathrm{Kpa})$. The pressures at the Viking Lander 2 site were 7.3 and 10.8 millibars. In comparison, the average pressure on Earth is 1000 millibars. Therefore, the material of the nanorobots has to be strong enough to sustain the internal force generated

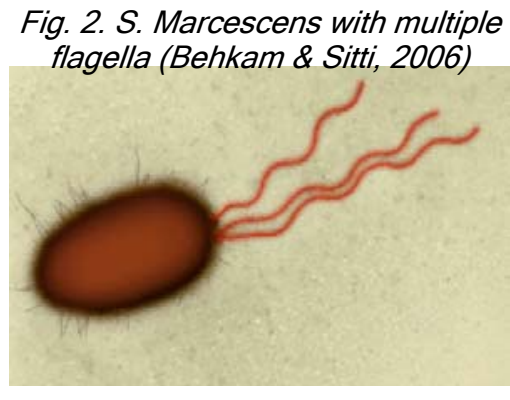

Fig. 2. S. Marcescens with multiple

"Nanorobot in Mars's mission" http://www.indjst.org
Vol.2 No. 8 (Aug 2009)

ISSN: 0974- 6846 due to the higher inside pressure and the designed nanorobots must be capable of surviving in such low pressures as on the martian surface.

Repair sequence

The important aspects of this particular architecture for sealing the breach are as follows:

- Mars suit repair nanorobots will be a sandwiching layer between the upper layer exposed to the external environment and the lower layer retaining oxygen at a pressure of $560 \mathrm{Kpa}$.

- Since the pressure on the Martian surface is very low (0.7 Kpa) (Hamilton 2001), when a region of the suit is damaged, the nanorobots will immediately rush to that region due to the pressure difference.

- Coordinated movement of the nanorobots, to the region of damage based on a set of behavioral rules, is ensured by the information gradients established by the contact signals (chemicals released by the nanorobots).

- Once they reach the damage, nanorobots quickly seal breaches by interlocking with one another in successive waves until the damage is sealed.

- This interlocking process of the nanorobots will follow a particular bonding sequence so that the breach is sealed in a very fast and efficient manner.

\section{Nanorobot design features}

The proposed nanorobot is required to perform a set of pre-defined tasks at the time of suit damage. Taking into account the Martian environmental conditions, the functionality of the nanorobots will be discussed with respect to the following features: Propulsion mechanism, swarm intelligence, behavioral strategy, behavioral rules and communication.

Propulsion mechanism

The nanorobot is entitled to have 6 degrees of freedom, so that it is not restrained from moving or rotating in any direction as it moves towards the damage. With regards to this, the propulsion mechanism of nanorobot is inspired by the flagellar mechanism of bacteria, in particular a bacterium named Serratius marcescens (Behkam \& Sitti, 2006). This bacterium is chosen because of its ability to reach high speeds in a very short time with almost $100 \%$ efficiency. The flagellum is driven by a rotary engine which is located in the anchor point on the inner edge of the nanorobot. The rotor alone can operate at 6000 to $17000 \mathrm{rpm}$ but with the filament attached it can reach only 200 to $1000 \mathrm{rpm}$. Flagella do not rotate at constant speed but can increase or decrease their speed depending on the proton motive force. With this mechanism the bacteria moves through the fluid (Fig. 2) at a speed of 60 cell lengths/sec which is much faster compared to cheetah which can reach a maximum of only 25 body lengths/sec (110 $\mathrm{km} / \mathrm{hr}$ ) (Behkam \& Sitti, 2006).

Another possible approach here could be to isolate the flagellar motor from a 
bacterium and to integrate it with the body of the nanorobot thereby reducing the complexities involved in fabrication and integration of artificial flagella. This stream of engineering is referred to as the nanochimeric engineering as suggested by Gajendran (2007).

We propose that the motor in the nanorobot is powered by electric potentials in the form of pulses produced by mechano-electrical transducers. When the nanorobots collide with the surrounding entities, torque is generated between a stator connected to the rigid anchor wall and a rotor connected to the filament. Electric impulses cause the stator to change shape or to move which exerts a force on the rotor and thereby causing it to rotate. In each cycle the rotor advances by one or two steps with equal angular increment. In the case of bacterial flagellar motor it has been found that there are about 50 steps/revolution per stator element and the rotation is not that smooth (Behkam \& Sitti, 2006). But the intact motor with 8 stator elements produces a smoother revolution with about 400 steps/revolution. The kinematics of the nanorobot, primarily governed by its motor dynamics, is modeled entirely based on the flagellar mechanism of $S$. marcescens.

\section{Viscosity vs. Inertia}

Flagellar mechanics is governed by viscosity and not by inertia due to the low Reynolds number regime of operation $(R e<<1)$. Here the propulsion force is due to the viscous drag. A thin rod experiences twice as great a force when it is moved sideways as when moved length wise (Fig.2). A slanting rod when pulled sideways immediately tries to become horizontal. With regards to this, when the rotor rotates the helical filament, it can be visualized as pushing and twisting a sequence of slant rods. Since each slant rod tries to become horizontal, a net force is exerted which pushes the nanorobot forward. The shape of the nanorobot can be approximated to closely fit into a sphere. This approximation is made to facilitate less intensive computations. The diffusion length $L_{D}$ for a small spherical particle is computed as:

$$
L_{d}=(4 D t)^{1 / 2}
$$

where $D$ is the diffusion coefficient and $t$ is the time.

$$
D=\frac{k_{B} T}{6 \Pi \eta R}
$$

$k_{B}$ is the Boltzman's constant, $T$ is the absolute temperature, $\eta$ is the coefficient of viscosity of the medium and $R$ is the radius of the particle.

The viscous drag is given by $F_{\text {drag }}=6 \Pi \eta R U$ -

for low Reynolds number $R_{e}<<1$ (where $U$ is the velocity of nanorobot).

The illustration to this propulsion mechanism explained before (Behkam, 2007) wherein latex beads of $400 \mathrm{~nm}$ diameter were propelled by a number of bacterial flagella attached to its surface. The net displacement was observed to be $90 \mu \mathrm{m}$, whereas the displacement due to Brownian motion was found to be only $0.9 \mu \mathrm{m}$ (hundred
Vol.2 No. 8 (Aug 2009)

ISSN: 0974- 6846 times smaller), hence proving that the beads were actually displaced by the flagella motors and not by Brownian motion.

\section{Swarm intelligence}

Swarm intelligence, a subset of multiagent systems approach is about teams of a number of units working together to achieve a common goal (Les Gasser et al., 1989). The units could eventually be engineered to nano size in numbers of tens, hundreds or even thousands (Cavalcanti \& Freitas Jr., 2005; Galstyan et al., 2005). Because of their scale and dynamics it makes it impossible to have a centralized coordination and global sharing of state. Therefore, schemes that can bring about inherently distributed behavior based on localized inputs, algorithms and outputs are required.

In this case of Mars suit repair the common goal will be to effectively seal the damage as quickly as possible. Once the nanorobots reach the damage they should work in a collective fashion so that they are able to seal the damage in successive concentric waves. This will require the nanorobots to communicate each other by sending signals that will propagate through network of nanorobots and set up a network hop count gradient. Information gradient is established in the form of chemical gradients (Wasielewski et al., 1997), set up by the guide post nanorobots. The nanorobots from other places will be attracted to this emergency zone by the information gradient. Once the nanorobots reach the damage they must execute a particular bonding sequence to seal the damage.

In this context it is required for the nanorobots to have a 'decision making mechanism' (Galstyan et al., 2005). In multiple simultaneous damages, all the nanorobots from that zone should not travel to another emergency zone as it can weaken the sealing process in that zone. Those nanorobots which were not involved in the previous task will send a signal through the network indicating that they are ready for the next repair. All these signals are encoded based on their intensity and the relative hop count with respect to each other and is made available to each nanorobot by means of entangled communication without involving any complex computation. A comparison among them is executed and only those nanorobots which have close proximity to the emergency zone will go for the repair and others will reorganize themselves for the next emergency.

There are several biological examples of colonies of individual agents (which behave in a similar way) with very limited computing ability yet bringing about an intelligent self organization. The colony of ants, birds, fishes and neuritis are some of the examples (Les Gasser et al., 1989). Based on the study of some of the swarm behaviors observed in nature the nanorobots are devised to work based on the following behavioral strategy.

\section{Behavioral strategy}

Previous approach towards this work was done by Gasser on distributed Artificial Intelligence (Les Gasser et 
al., 1989), in which the problem was divided into a number of phases: 1) Problem recognition, 2) Enlisting allies, 3) Formation of a coordination network, 4) Midgame problem, 5) End game problem solving, and 6) Termination.

Therefore in order to implement a real time coordinated swarm action it is necessary to define a set of behavioral rules which will govern the behavior of each nanorobot.

Behavioral strategy will be as follows:

- directional control produced by chemical (information gradients)

- movement induced by the pressure difference and aided by the flagellar motion

- guidance by various guidepost or pioneer units that usher other units to proper location

- induced competition among the nanorobots of the same unit

Behavioral rules: In accord with the behavioral strategy a reactive architecture is used i.e. percepts of information gradients (chemical signals) will cause a behavior. Behaviors with higher precedence can suppress the expression of other behaviors. This is in a style similar to the architecture proposed and implemented by Brooks (Rodney Brooks, 1986).

The behavior of each nanorobot is decided by a simple set of reactive rules. These rules determine the behavior of nanorobot at any instant. The rule selection is based on the information input and the precedence of the rule. These rules can be implemented as non deterministic rule based system and the rule predicates are evaluated in sequential order. When a rule's predicate is satisfied the action described by the rule is instantiated.

Info gradient. The first few nanorobots (also known as the guide post units) which reach the region of damage primarily due to the pressure difference will establish a chemical gradient by the release of suitable chemicals. Chemical concentration $C$ (in $\mu^{3}$ ) at any point $(\mathrm{x}, \mathrm{y}, \mathrm{z}$ ) with the chemical source at origin, is given by:

$$
C(x, y, z)=\frac{Q}{2 \Pi D r} e^{-v(r-x)(2 D)}
$$

where $Q$ is the rate at which the chemical is produced by its point source; $D$ is the diffusion coefficient of the chemical and $r$ is the radial distance from the point source (Berg, 1993). This chemical concentration will in turn establish an Information (INFO) gradient depending on the magnitude of the concentration at various points on the trajectory of the guidepost nanorobot.

Threshold: The nanorobots will be designed in such a way that they have a minimum limit on their sensitivity. Therefore the nanorobots will not be able to detect INFO (chemical) Gradients with magnitudes less than a certain Threshold magnitude which is decided by the design specifications of their sensory units.
The set of rules which the nanorobot execute is called its program rule set. The program rule set is for a specific condition, during a specific time. The program rule set for the nanorobots is as follows:

Rule l: IF (NO (INFO MARKER) AND NO TEAR) THEN (random walk))

Rule If: IF (TEAR) THEN (bond sequentially) AND Differentiate AND Broadcast

Rule III: IF (Magnitude (INFO) < Threshold) THEN (random walk)

Rule IV: IF (DETECT INFO) THEN (move up INFO Gradient) OR (Differentiate) AND (Broadcast)

The above mentioned rules are applicable only till the nanorobots reach the region of damage after which they follow a set of bonding rules which are discussed in detail in Phase 2 of the following section.

Modeling the communication between nanorobots: This modeling approach is divided into two phases: Phase 1: Modeling the communication among the nanorobots till they reach the region of damage. Phase 2: Modeling the interaction among the nanorobots during the interlocking mechanism to seal the damage. A similar approach of interlocking mechanism has been proposed in (Benjamin Chui \& Lea Kissner, 2000), but investigation on the propulsion mechanism of the nanorobot has not been dealt in detail which indeed has been well explained and illustrated in this paper.

Phase 1:

Phase 1 deals with the trajectory of the nanorobots from their initial position to the region of damage. To reduce the computational complexity the whole process was simulated in a 2D space with the velocity and position vectors of the $i^{\text {th }}$ nanorobot denoted as:

$$
\begin{aligned}
& \mathbf{V}_{\mathbf{i}}=\left[\begin{array}{ll}
\mathrm{v}_{\mathrm{ix}} & \mathrm{v}_{\mathrm{iy}}
\end{array}\right]^{\top} \\
& \mathbf{X}_{\mathbf{i}}=\left[\begin{array}{ll}
\mathrm{x}_{\mathrm{i}} & \mathrm{y}_{\mathrm{i}}
\end{array}\right]^{\top}
\end{aligned}
$$

At the end of every time step of the simulation the velocity and position vectors were updated based on the following iterative equations-

$$
\begin{aligned}
\mathrm{V}_{\mathrm{i}}(\mathrm{t}+1)= & \mathrm{A} * \mathrm{~V}_{\mathrm{i}}(\mathrm{t})+\mathrm{R}_{1} *(\Delta \mathrm{t})^{-1} *\left(\mathrm{X}_{\mathrm{i}}(\mathrm{t}-1)-\mathrm{X}_{\mathrm{i}}(\mathrm{t})\right)-(7) \\
& \mathrm{X}_{\mathrm{i}}(\mathrm{t}+1)=\mathrm{X}_{\mathrm{i}}(\mathrm{t})+(\Delta \mathrm{t}) * \mathrm{~V}_{\mathrm{i}}(\mathrm{t}+1)
\end{aligned}
$$

where $A$ represents the acceleration factor, $R_{1}$ and $R_{2}$ are the ratcheting factors, $\Delta$ t denotes the time step in simulation and $X_{D}$ represents the approximated position of the damage $D$.

$A, R_{1}$ and $R_{2}$ are dynamic vectors which are computed at the end of every time step before updating the velocity and position vectors. Acceleration factor A depends on the pressure difference and the concentration gradient at that instant, hence it is defined in the following way-

$A=f(\Delta P(x, y, t), \Delta C(x, y))$

where $f$ is the defining function, $\Delta C$ represents the 2-D (two dimensional) concentration gradient (INFO gradient) at a particular $(x, y, z=0)$ with respect to a reference 
(damage region $D$ ) and $\Delta P$ denotes the pressure difference with respect to the reference at a given instant.

Ratchets: The most hindering factor to the coordinated movement of the nanorobots is the thermal noise. Thermal vibrations which are triggered by the heat exchanges taking place in the nano-scale environment tend to cause a drastic deviation in the trajectory of the nanorobots. To nullify the effect of these vibrations, the concept of thermal ratchets was adopted (Ajdari \& Prost, 1992; Magnasco,1993) (Fig.3). A ratchet is a rectifying structure where movement is easier in one direction than the other because a larger force is necessary for one direction compared to the other (Fig.4). This type of ratchet uses an oscillating time-averaged zero force as its input (e.g. an Efield or a pressure gradient), which it rectifies (Ajdari \& Prost 1992).

Though the rectifying ratchets are not physically shown in the simulation, their effect is modeled by introducing the ratcheting factors in the velocity vector updating equation. Ratcheting factors $R_{1}$ and $R_{2}$ depend on the specifications of the ratchet and the instantaneous pressure difference at that location. Hence ratcheting factors are defined as follows:

$$
\begin{aligned}
& R_{1}=h\left(r_{1}(x, y), \Delta P(x, y, t)\right) \\
& R_{2}=h\left(r_{2}(x, y), \Delta P(x, y, t)\right)
\end{aligned}
$$

where $h$ denotes the defining function, $r_{1}$ and $r_{2}$ are the ratcheting constants and $\Delta P$ denotes the pressure difference with respect to reference (damage region $D$ ) for a given instant.

Phase 2:

As soon as the nanorobots reach the damage they should follow a highly efficient interlocking mechanism such that the violently out gassing damage is sealed in the shortest time duration possible. The nanorobots will have the necessary inbuilt sensor to detect the worn out portion of the suit as soon as it reaches the outer edge of the tear. The first set of nanorobots which reach the tear will immediately bond to the molecules that form the outer edge of the suit fabric. This bonding will be initiated because of the van der waals attractive forces between

Popular article

CIndian Society for Education and Environment (iSee)

the suit molecules and the nanorobots. By this bonding the first wave of nanorobots will form a barrier against the escaping gas on the outer edge of the damage.

To ensure quick sealing, a major portion of the nanorobot should resemble a cube with the flagella at the bottom for their propulsion. The nanorobots which formed the initial barrier on the outer edge of the damage will have a design feature such that they induce a contact signal on their faces opposite to the ones that have been bonded to the suit fabric. Therefore the nanorobots arriving at the damage in the next successive wave will bond only onto the face with the induced contact signal. This particular interlocking mechanism is to ensure that they repair more or less aligned with the damage and can converge to seal the tear quickly. The successive interlocking by the nanorobots by inducing the contact signals can be modeled as Van der waals interactions (Peng et al., 1997).

Van der waals interactions

Due to the neutral nature of both the fabric material and the nanorobots we make use of van der Waals interactions because they are a source of short range interactions produced by the London forces arising from the induced dipole interactions (here the contact signal) (Peng et al., 1997, Cornell et al., 1995).

Short range interactions offer the possibility to take into account only neighboring particles up to a certain distance for the calculation of interactions. In that way a cutoff radius is introduced beyond of which the mutual interactions between particles are neglected. As an approximation one may introduce long range corrections to the potential in order to compensate for the neglect of explicit calculations. The whole short range potential

Fig.4. Comparison between the Buckingham potential, the Lennard-Jones (12-6) \& Lennard-Jones (9-6) potentials (Peng et al., 1997; Cornell et al., 1995)

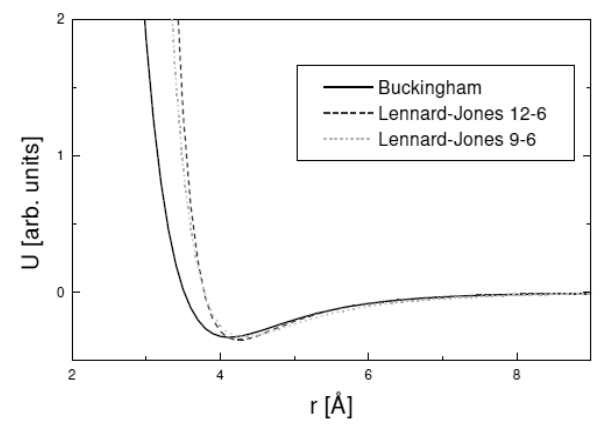

(Cornell et al., 1995), may then be written as:

$$
U=\sum_{i<j}^{N} u\left(r_{i j} \mid r_{i j}<R_{c}\right)+U_{l r c}
$$

where $R_{c}$ is the cut-off radius and $U_{l r c}$ is the long range correction factor.

The long-range correction, is given as

$$
U_{l r c}=2 \pi N \rho_{0} \int_{R_{c}}^{\infty} d r r^{2} g(r) u(r)
$$

Where $\rho_{0}$ is the number density of the particles in the system and $g(r)=\rho(r)=$ $\rho_{0}$ is the radial distribution function. For computational reasons, $\mathrm{g}(\mathrm{r})$ is most often only calculated up to $R_{c}$ so that in practice it is assumed that $\mathrm{g}(\mathrm{r})=1$ 
for $r>R_{c}$, which makes it possible for many types of potentials to calculate analytically (Cornell et al., 1995). Here we consider a very special case of the short range interactions - the van der waals interactions. The instantaneously created dipoles induce dipoles on the faces of the nanorobots that arrive in the next successive wave.

Two common forms of the resulting interactions are:

1) Buckingham potential (Peng et al., 1997; Cornell et al., 1995).

$$
u_{\alpha \beta}^{B}\left(r_{i j}\right)=A_{\alpha \beta} e^{-B_{\alpha \beta} r_{i j}}-\frac{\check{D_{\alpha \beta}}}{r_{i j}^{6}}
$$

2) Lennard-Jones potential (Peng et al., 1997; Cornell et al., 1995):

$$
u_{\alpha \beta}^{L J}\left(r_{i j}\right)=4 \epsilon_{\alpha \beta}\left(\left(\frac{\sigma_{\alpha \beta}}{r_{i j}}\right)^{12}-\left(\frac{\sigma_{\alpha \beta}}{r_{i j}}\right)^{6}\right)
$$

In Eqs.14,15 the indices $\alpha, \beta$ indicate the species of the particles, i.e. there are parameters $A ; B ; D$ in Eq.14 and $\varepsilon, \sigma$ in Eq.15. For intra-species interactions $(\alpha=\beta)$ as in the case of interaction between the nanorobots and for cross species interactions $(\alpha \neq \beta)$ as in the case of interaction between the nanorobots and the molecules of the suit fabric.

For the Lennard-Jones potential the parameters have a simple physical interpretation: $\varepsilon$ is the minimum potential energy, located at $r=2^{1 / 6} \sigma$ and $\sigma$ is the diameter of the particle, since for $r<\sigma$ the potential becomes repulsive. Often the Lennard-Jones potential gives a reasonable approximation of a true potential. Since computationally the Lennard-Jones interaction is quite attractive the repulsive part is sometimes replaced by a weaker repulsive term $r^{1 / 9}$ instead of $r^{1 / 12}$. The Fig. 4 provides a comparison between the Buckingham potential, the Lennard-Jones (12-6) and Lennard-Jones (9-6) potentials.

The Lennard-Jones potential has another advantage over the Buckingham potential, since there are combining rules for the parameters. A common choice is the Lorentz-Berelot combining rules Nymand et al., 2000. $\sigma_{\alpha \beta}=\frac{\sigma_{\alpha \alpha}+\sigma_{\beta \beta}}{2} \quad, \quad \epsilon_{\alpha \beta}=\sqrt{\epsilon_{\alpha \alpha} \epsilon_{\beta \beta}}$

In our modeling approach, the LennardJones (9-6) potentials are used because their comparable accuracy (as evident from the graph) and computationally less intensive nature as compared to the original Lennard-Jones (12-6) potentials because of their more attractive and less Popular article

CIndian Society for Education and Environment (iSee)

repulsive nature (Cornell et al., 1995).

With the help of our modeling approach the system potential $U$ was computed in every time step of the simulation and only that configuration with the least potential energy was accepted. This least energy configuration was found to be similar to the bonding that we had proposed - Nanorobots bonding onto the face with the induced contact signal of the already bonded nanorobots. Since the forces involved in the interactions vary as: $F=-\nabla U-(17)$

at every time step the

Fig. 5 a) Colony of nanorobots on either side of the tear caused at

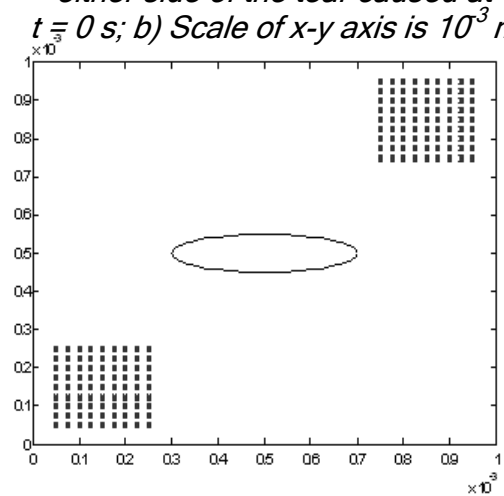

Fig. 6 a) Nanorobot colony at $t=10 \mathrm{~ms}$. b) Nanorobots are propelling towards the tear \& some have bonded to its outer edge. c) Scale of $x-y$ axis is $10^{3} \mathrm{~m}$

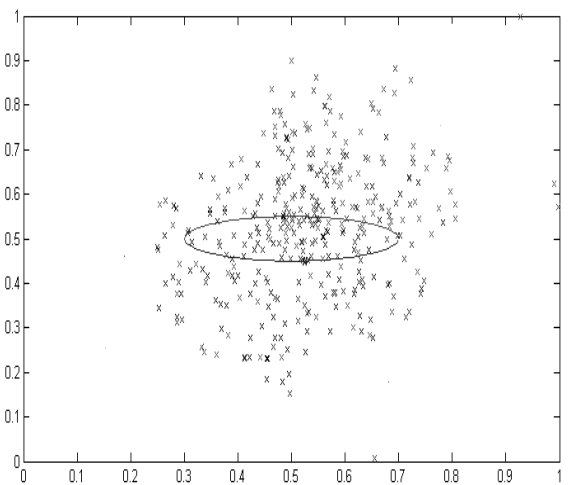

Fig. 7a) Nanorobot colony at $t=20 \mathrm{~ms}$. b) Portion of the damage is sealed by adopting the interlocking mechanism. c) Scale of $x-y$ axis is $10^{3} \mathrm{~m}$

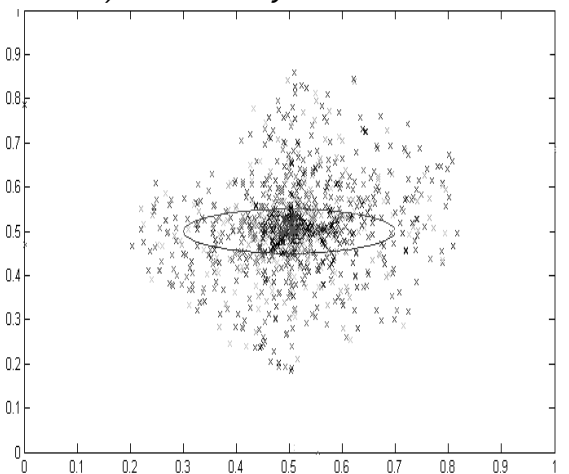

"Nanorobot in Mars's mission" http://www.indjst.org forces acting on the nanorobots were determined and their corresponding velocity vectors were modified accordingly.

Simulation in 2- D space (Fig.5-10)

One particular situation wherein the mars suit was subjected to a tear of length $0.5 \mathrm{~mm}$ and width $0.1 \mathrm{~mm}$ was considered and a simulation was performed based on the equations modeled for the behavioral rules of Phase / and the bonding sequence rules of Phase II. The following results were obtained for different instances of the simulation. The tear was assumed to be of elliptical shape with its centre at $(0.5,0.5)$ as shown in the Fig. 5. The simulation results clearly demonstrate that the sealing of the tear happens in a fast and efficient manner because of the collective action of nanorobots thereby validating the solution proposed in this paper.

\section{Conclusions}

The concept of a real time intelligent system consisting of a colony of nanorobots functioning in a coordinated manner to seal the

damage caused on the suit of the astronaut involved in field exploration on mars has been proposed. It provides a possible, cost and time effective solution to quickly seal the damage so that the astronaut has enough time to return to the pressurized habitat safely. A simulation was carried out to demonstrate the efficiency of swarm action i.e. the distributed behavior of nanorobots achieved by a set of behavioral rules and bonding sequence rules with the common goal of reaching the damage and sealing it quickly. 
Future work The recent advances in nanofabrication technology promises a number of breakthroughs in the structural and assembly aspects of nanorobotic engineering. One particular example is the fabrication of artificial bacterial flagella propelled by an external magnetic field (Zhang et al., 2009) thereby demonstrating the feasibility of artificial propellers for any application requiring the deployment of nanorobots. Since the artificial flagella are externally propelled, it makes them inadequate for real time medical applications. Hence, engineering an untethered nanomotor powered by onboard power supply or by deriving energy from the surrounding medium would be one of the greatest breakthroughs of nanorobotics for medical applications, thereby enabling their deployment in any part of the body for highly sophisticated disease detection and effective treatment in real time.

Acknowledgements The author is grateful to Dr. Milind Pimprikar (Founder of CANEUS organization and President of Centre for Large Space Structures and Systems Inc., Canada) for having provided an opportunity to present the ideas and initial stages of this work in NASA AMES Research Center (California, USA) during the Caneus 2009 Workshops - International Collaborative Aerospace Development with a special focus on Micro \& Nanotechnologies. The author also acknowledges Prof. Kameshwar Rao for his input and feedback on this work.

\section{References}

1. Adriano Cavalcanti, Waren W, Wood, Luiz C, Kretly and Bijan Shirinzadeh (2006) Computational Nanomechatronics: A Pathway for Control and Manufacturing Nanorobots. IEEE CIMCA Intl. Conf. on Computational Intelligence for Modelling, Control \& Automation. IEEE Computer Society, Sydney, Australia www.nanorobotdesign.com.

2. Ajdari A and Prost J (1992) Drift induced by a spatially periodic potential of low symmetry: pulsed dielectrophoresis. CR Acad. Sci. Ser. II. 315,16351639.

3. Behkam B and Sitti M (2006) Design methodology for biomimetic propulsion of miniature swimming robots. ASME J. Dynamic Sys. Measur. Control. 128 (1), 3643.

4. Behkam B and Sitti M (2007) Bacterial Flagella based propulsion and on/off motion control of microscale objects. Applied Physics Letters. 90(2): 1-3.
Vol.2 No. 8 (Aug 2009)

ISSN: 0974- 6846
Fig. 8 a) Nanorobot colony at $t=30 \mathrm{~ms}$. Major portion of the damage has been c) Scale of $x-y$ axis is $10^{3} \mathrm{~m}$

Fig. 9. Schematic diagram of the nanorobot with its 2 main units sensors and actuators and (ii) helical tail

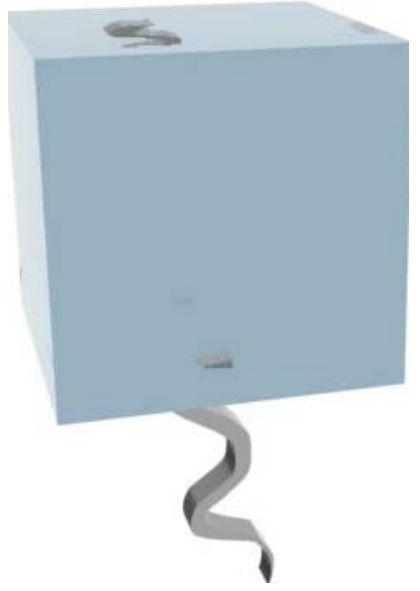

5. Benjamin Chui and Lea Kissner (2000) Nanorobots for Mars EVA repair. Soc. Auto. Engineers, Inc.

6. Berg HC (1993) Random walks in biology. Princeton Univ. Press, $2^{\text {nd }}$ edition.

7. Carslaw RS, Harrison RG and Kirkby J (2002) Cosmic rays, clouds and climate. Science. 298, 1732.

8. Cavalcanti A (2003) Assembly automation with evolutionary nanorobots and sensor-based control applied to nanomedicine. IEEE Trans. on Nanotech. 2 (2), 82-87.

9. Cavalcanti A and Freitas Jr. RA (2005) Nanorobotics control design: A collective behavior approach for medicine IEEE Trans. on NanoBioSci. 4(2), 133-140.

10. Cavalcanti A, Warren W Wood, Louiz K Kretly, Bijan Shirinzadeh (2006) Computational nanomechatronics: A pathway for control and manufacturing nanorobots. IEEE CIMCA International conference on computer intelligence for modeling, control and automation, IEEE computer society, Sydney, Australia.

11. Cornell WD, Cieplak P, Bayly Cl, Gould IR, Merz KM, Ferguson DM, Spellmeyer DC, Fox T, Caldwell JW and Kollman PA (1995) A second generation force field for the simulation of proteins, nucleic acids, and organic molecules. J. Am. Chem. Soc. 117, 5179-5197.

12.Couvreur $P$ and Vauthier $C$ (2006) Nanotechnology: intelligent design to treat complex disease. Pharmaceut. Res. 23 (7), 1417- 1450.

13. Freitas RA Jr. (1997) Basic capabilities Nanomedicine vol. I. Landes Bioscience.

14.Freitas RA Jr. (2003) Biocompatibility -Nanomedicine, vol. II, Landes Bioscience.

15.Gajendran N (2007) Adding Life to the nanotechnology. Indian J. Sci. Technol. 1 (1), 1-5. Domain:http://www.indjst.org.

16. Galstyan A, Hogg T and Lerman K (2005) Modeling and mathematical analysis of swarms of microscopic robots IEEE Swarm Intelligence Sym. Pasadena CA, USA. pp:201-208.

17. Grant JA, Arvidson R, Bell III JF and Cabrol NA (2004) Surficial deposits at Gusev Crater along Spirit Rover traverses. Science; Aug 6,305, 5685, p: 807. 
18. Greeley R, Squyres SW, Barlett $P$ and Banley D (2004) Wind related processes detected by the Spirit Rover at Gusev Crater, Mars. Science Aug 6, 305, 5685, p: 910.

19. Hamilton CJ (2001) Mars Introduction. http://www.solarviews.com/eng/mars.htm.

20. Hoffman, Stephen J and David L NASA (1997) Mars exploration study team. Human exploration of Mars. The reference mission of the NASA Mars exploration study team. Kaplan ed. vol.7.April1999.

21. Howard J (1997) Molecular motors structural adaptations to cellular functions. Nature. 389, 561567.

22. Leary SP, Liu CY and Apuzzo MLI (2006) Toward the emergence of nanoneurosurgery: Part III Nanomedicine: Targeted nanotherapy, nanosurgery, and progress toward the realization of nanoneurosurgery. Neurosurgery. 58 (6), 1009-1025.

23. Les Gasser, Nicholas F, Rouquette, Randall W, Hill and John Lieb (1989) Representing and using organizational knowledge in distributed Al systems. Distributed Artificial Intelligence. 2, 55-78. Morgan Kaufmann Publ., Inc, San Mateo, CA.

24.Li W, Xi JN, Fung WK and Wong TS (2004) Nanorobotics and Nanomanipulation. Encyclopedia of Nanosci. Nanotechnol., Am. Sci. Publ. 7(15), 351365.

25. Magnasco MO (1993) Forced thermal ratchets. Phys. Rev. Lett. 71(10), 1477-1481.

26. Milam KA, Stockstill KR, Moersch JE, McSween, Jr. HY, Tornabene LL, Ghosh A, Wyatt MB, and Christensen PR, THEMIS characterization of the MER Gusev crater landing site, J. Geophys. Res., $108,8078$.

27. Montemagno C and Bachand G (1999) Constructing nanomechanical devices powered by biomolecular motors. Nanotechnol. 10, 225 - 231.

28. Morris K (2001) Macrodoctor come meet the nanodoctors. The Lancet. 357,778.

29. Nymand TM, Linse P and Ewald (2000) Summation and reaction field methods for potentials with atomic charges, dipoles and polarizabilities J. Chem. Phys. 112, 6152 - 6160.

30.Patel GM, Patel GC, Patel RB, Patel JK and Patel M (2006) Nanorobot: A versatile tool in nanomedicine. J. Drug Targeting 14 (2), 63-67.

31.Peng ZW, Ewig CS, Hwang MJ, Waldman $M$ and Hagler AT (1997) Derivation of class ii force fields. 4. van der Waals parameters of alkali metal cations and halide anions. J. Phys. Chem. 101,7243 - 7252.

32. Rodney Brooks (1986) A layered intelligent control system for a mobile robot. IEEE J. Robotics \& Automation RA-2, 14-23.

33. Squyres SW, Arvidson RE, Bell III JF and Bruckner J (2004) Spirit Rover's Athena science investigation at Gusev crater, Mars. Science. 305, 5685; p: 974.
34.Svensmark H, Friis-Christensen E (1997) Variation of cosmic ray flux and global cloud coverage - A missing link in solar - climate relationships. J. Atmos. Terr. Phys. 59, 1225.

35. Toth-Fejel T (2000) Agents, assemblers, and ANTS: scheduling assembly with market and biological software mechanisms. Nanotechnol. 11, 133-137.

36. Wasielewski R, Rhein A, Werner M, Scheumann GF, Dralle H, Potter E, Brabant G and Georgii A (1997) Immunohistochemical detection of Ecadherin in differentiated thyroid carcinomas correlates with clinical outcome. Cancer Res. 57(12), 2501-2507.

37.Weiss R and Knight Jr.TF (2000) Engineered communications for microbial robotics. Proc. of 6th Intl. Meeting on DNA Based Computers (DNA6).

38. Yu F (2002) Altitude variations of cosmic ray induced production of aerosols: Implications for global cloudiness and climate. Journal of Geophysics. Res. 107, A7; Published online 19 July (10.1029/2001 JA0D0248).

39.Zhang MJ, Sabharwal CL, Tao W, Tarn TJ, Xi N and Li G (2004) Interactive DNA sequence and structure design for DNA nanoapplications. IEEE Trans. on Nanobiosci. 3(4), 286-292.

Fig. 10. Schematic diagram of the nanorobot, showing the inner structural details. The spherical structure will house the units that induce the contact signal and the nanomotor will be housed in the cylindrical rod to which the helical filament will integrated

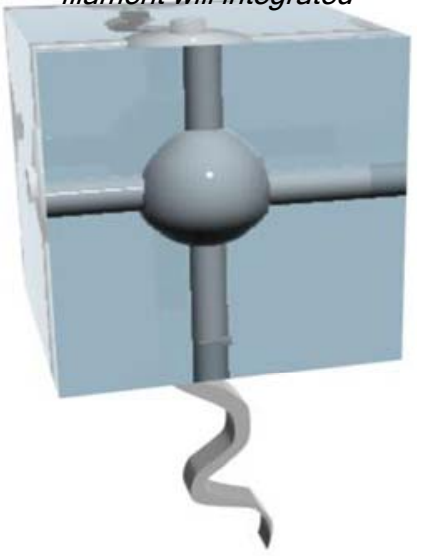

Popular article

CIndian Society for Education and Environment (iSee)
"Nanorobot in Mars's mission" http://www.indjst.org
Gautham

Indian J.Sci.Technol. 